\title{
Effect of microwave and conventional regeneration on the microporous and mesoporous network and on the adsorptive capacity of activated carbons
}

\author{
CO Ania, JB Parra, JA Menéndez, JJ Pis* \\ Instituto Nacional del Carbón, CSIC, Apartado 73, 33080 Oviedo, Spain
}

\begin{abstract}
The effect of different heating mechanisms on the porous network of activated carbons (AC) previously exhausted with phenol has been studied. To this end, thermal treatment of the exhausted AC was performed using two experimental devices: a single mode microwave device operating at $2450 \mathrm{MHz}$ and a conventional electric furnace. By employing microwave energy, the regeneration time was considerably shortened compared to conventional thermal heating. Moreover, microwave heating preserved the porous structure of the regenerated AC more efficiently than treatment in a conventional device. In both cases successive regeneration cycles reduced the microporosity of the activated carbons. However, conventional heating shifted the micropore size distribution to pores of narrower sizes. The apparent BET surface areas were also reduced significantly over the regeneration cycles. A loss of the adsorptive capacity of the carbon material was observed after six adsorption-desorption cycles in both systems. The phenol adsorption capacities decreased to a greater extent in the samples regenerated in the electric furnace.
\end{abstract}

Keywords: Activated carbon; Thermal regeneration; Microwave energy; Phenol adsorption; Carbon deposits 


\section{INTRODUCTION}

Over the last few decades, activated carbons (AC) have been employed in a wide number of applications on an industrial scale, including technologies for the purification of gases [1], and the removal of organic pollutants from water (i.e., purification of drinking water, and wastewater) [2-4]. Depending on their adsorption capacity, they become saturated after sustained use. Formerly, when an AC reached its saturation limit, it was usually taken to a landfill and dumped. However, the enclosing and burying of hazardous waste is becoming increasingly unacceptable due to growing concern about the effect of pollutants on the environment and more restrictive environmental regulations.

Efficient regeneration systems are required to permit a wider application of carbon adsorption processes and to ensure their economic feasibility [5]. This has motivated companies to develop methods for regenerating and reusing saturated activated carbon. Over the years, a wide variety of regeneration techniques have been suggested and applied. These are based either on desorption -induced by increasing the temperature or by displacement with solvents-, or on decomposition induced by thermal, chemical, catalytic or microbiological processes [6,7]. By far the most extensively used technique is thermal regeneration under steam or an inert atmosphere. Unfortunately, the regeneration process is time consuming and after successive heating and cooling cycles, the carbon becomes damaged.

The application of microwave-heating technology for regenerating industrial waste activated carbon has been investigated [8-10], with very promising results. The main difference between microwave devices and conventional heating systems is in the way the heat is generated. Thermal regeneration is conventionally performed in rotary kilns or vertical furnaces, where the carbon bed is indirectly heated by conduction and convection. In the microwave device, the microwaves supply energy directly to the carbon bed. Energy transfer is not by conduction or convection as in conventional heating, but is readily transformed into heat inside the particles by dipole rotation and ionic conduction [11]. When high frequency voltages are applied to a material, the response of the molecules with a permanent dipole moment or induced dipole to the applied potential field is to change their orientation in the direction opposite to that of the applied field. The synchronized agitation of molecules then generates heat.

Microwave regeneration offers possible advantages over conventional treatment. These include the rapid and precise control of the carbon bed temperature, a more compact furnace, and energy savings. Some authors have also found that microwave regeneration gives rise to a better performance of the activated carbons in terms of ulterior adsorption capacity and rate of adsorption compared to conventional heating [10, 12-14]. Consequently, processes based on microwave energy are gaining importance in several fields of application on an industrial scale, and microwave 
technology is now considered a promising available technology for the regeneration of carbon materials $[15,16]$.

The aim of this work was to acquire a deeper insight into the thermal regeneration of activated carbons. To this end a comparative study of the regeneration of activated carbons by conventional thermal methods and single mode microwave energy was performed. Changes in the porous texture of the regenerated carbons resulting from the thermal treatment in both devices were studied in relation to the changes in the load capacity of the adsorbents.

\section{EXPERIMENTAL}

\subsection{Materials}

According to the manufacturer, the selected activated carbons were obtained from the physical activation of coal (Q) and chemical activation of wood (CV) using phosphoric acid. Sample Q was ground and a particle size fraction of $0.212-0.075 \mathrm{~mm}$ was used in all the experiments. The second carbon sample, CV, was supplied in powdered form. The as-received samples were washed several times with deionised boiling water, dried for 2 days at $110^{\circ} \mathrm{C}$ and stored in a desiccator until used.

\subsection{Regeneration in an electric furnace and a microwave device}

The samples were saturated with phenol using a stainless steel column of length $10 \mathrm{~cm}$ and internal diameter $1.5 \mathrm{~cm}$. All the experiments were conducted at an initial concentration of $2 \mathrm{~g} \mathrm{l}^{-1}$, and a flow rate of $4 \mathrm{ml} \mathrm{min}^{-1}$. The outlet concentration was continuously monitored by means of a UV-VIS spectrophotometer at the corresponding wavelength. The adsorptive capacities were obtained by integrating over the entire breakthrough curve. When the saturation point was reached, the exhausted AC was washed with distilled water for 2 hours to eliminate any excess of pollutant, and dried overnight in an oven at $110{ }^{\circ} \mathrm{C}$ to reduce the moisture content. Afterwards, the sub-samples of the exhausted AC were regenerated.

Regeneration of the exhausted AC was carried out in an electric furnace (EF) and in a single mode microwave device (MW). The resultant samples were labelled sample-EF or sample-MW, respectively. The exhausted samples were regenerated at $850{ }^{\circ} \mathrm{C}$ under an inert $\left(\mathrm{N}_{2}\right)$ atmosphere. The spent AC was placed in a vertical quartz reactor and purged with inert gas. The sample was then heated using a nitrogen flow rate of $10 \mathrm{ml} \mathrm{min}^{-1}$. The inert atmosphere was maintained during the heating up and cooling-down intervals.

The experimental equipment for the microwave regeneration of AC has been described elsewhere [17]. The temperature of the regeneration process was measured with an optical pyrometer. Samples will be denoted as the carbon samples followed by CiRi -Ci being the cycles of 
saturation and Ri the number of regenerations-, plus a reference to the experimental device. For instance, sample QC2R2 MW corresponds to Q activated carbon after two subsequent adsorption/ regeneration cycles in the microwave device. The effect of the heating mechanism in the absence of the adsorbate was also evaluated; the blanks will be denoted as the carbon samples followed by EF or MW.

\subsection{Textural and chemical characterisation}

Textural characterisation was carried out by measuring the $\mathrm{N}_{2}$ and $\mathrm{CO}_{2}$ adsorption isotherms at -196 and $0{ }^{\circ} \mathrm{C}$, respectively. Narrow microporosity (pore diameter $<0.7 \mathrm{~nm}$ ), medium-sized microporosity $(0.7<$ pore diameter $<2 \mathrm{~nm})$ and mesoporosity were evaluated by means of the DFT model [18] applied to the nitrogen adsorption isotherms. This subdivision of microporosity provided by the DFT method, although not in strict accordance with that established by IUPAC, is widely accepted [19].

The micropore size distribution was assessed from $\mathrm{CO}_{2}$ adsorption at $0{ }^{\circ} \mathrm{C}$ [20]. For this purpose, the DRS equation was also applied to the $\mathrm{CO}_{2}$ adsorption isotherms, the $\beta$ parameter being 0.36 [21] and the packing area for the $\mathrm{CO}_{2}$ molecule $0.187 \mathrm{~nm}^{2}$ [22].

The materials were further characterized by ultimate analysis and the determination of the point of zero charge. The PZC values were determined by mass-titration as described elsewhere [23].

\section{RESULTS AND DISCUSSION}

\subsection{Regeneration in the electric furnace and microwave device}

The exposure time of the activated carbon at the temperature of regeneration was set as the reaction time necessary to attain a $3 \%$ degree of burn-off under a $\mathrm{CO}_{2}$ atmosphere, in order to compare the results with those obtained under oxidizing conditions, as discussed in previous works [17]. This parameter was measured in both experimental devices. The time was found to be 9 times shorter when the samples were regenerated by means of microwave energy ( $4 \mathrm{~min}$ ) compared to the conventional EF (37 min). The time needed to rise from room temperature to $850{ }^{\circ} \mathrm{C}$ was also lower when microwave energy was applied. The AC reached the experimental temperature in 5-6 minutes when heated by microwave energy, whereas 10-13 minutes were necessary for the samples treated in the EF.

The influence of thermal treatment on the porous structure of non-saturated AC has been reported previously. In general, microwave heating of non-saturated activated carbon preserves the porous structure of the parent sample to a greater extent, than treatment carried out in the EF. 
Specific surface areas and micropore volumes in the samples treated in the MW did not differ noticeably from those of the parent AC. Whereas, changes were greater after treatment of the nonsaturated AC in the EF, giving rise to some blocking of the porous structure [17].

\section{a) Temperature uniformity in the microwave device}

Water has a high dielectric loss factor, so relatively small differences in moisture content between samples will result in different temperatures. To eliminate the changes in the strength of the electric fields in the material and the power dissipated as a consequence of variations in moisture content, the saturated samples were dried at $110^{\circ} \mathrm{C}$.

During microwave heating, the energy is applied directly into the sample. The temperature reached will not only depend on the fraction of the supplied energy absorbed by the sample but also on the nature of the AC (dielectric properties, degree of order of the basal planes, chemical composition, etc.) [24, 25], its particle size, and the amount of microwave power applied to the sample [11]. For a given AC, the temperature can be modified by adjusting the input power. In the present work, the temperature was fitted by using a variable input power, taking into account the requirements of each sample. The saturated AC reached the experimental temperature $\left(850^{\circ} \mathrm{C}\right)$ after 5-6 minutes, this temperature remaining practically stable during the treatment. In contrast about 14 minutes was needed when regeneration was conducted in the conventional electric equipment. The amount of carbon, moisture content and particle size were found to be approximately the same for all the assays.

When the exhausted carbons were exposed to the microwaves, the input power used to reach the regeneration temperature $\left(850^{\circ} \mathrm{C}\right)$ had to be increased with each regeneration cycle from 300 to 800 Watts (Figure 1). These differences are probably due to the changes induced in the microstructures of the carbon, and the saturation degree. Nevertheless, it was difficult to establish any correlation between the saturation cycles and the fraction of supplied energy that was adsorbed. After a series of adsorption/desorption cycles the changes induced in the chemical composition and/or porous structure of the regenerated AC may alter the dielectric properties of the adsorbents considerably. As a result the fraction of absorbed energy needed to reach the final temperature is also modified.

In most cases, heating the samples in the microwave device was quite fast, less than 6 minutes being needed to reach $850^{\circ} \mathrm{C}$, except for sample $\mathrm{CV}$ in the first regeneration cycle. In general, a rapid initial heating up to $140{ }^{\circ} \mathrm{C}$ was followed by a period during which volatiles were removed from the sample. There was then a rapid increase in temperature up to $850{ }^{\circ} \mathrm{C}$. Subsequent treatments of the exhausted activated carbons (i.e., a second and successive series of regeneration cycles) led to a steady increase in temperature. Desorption of the adsorbate retained on the activated carbon was observed after $200{ }^{\circ} \mathrm{C}$ (change in the colour of the evolved gases). 
The slow heating rate of the CV sample during the first regeneration cycle was also observed for the heating of the as-received sample (non-saturated). In this case, the period of gas removal at low temperatures was much longer, approximately 12 minutes being needed to reach $850{ }^{\circ} \mathrm{C}$. During this step, a large amount of gases was observed to evolve from the carbon bed, and the fraction of power absorbed by the activated carbon decreased suddenly. The microwaves applied to the carbon bed were not absorbed, and a considerable percentage of the energy was transmitted by the sample. This might be explained in terms of the different chemical composition of the two activated carbons, if it is assumed that some changes occur on the surface chemistry of the AC during microwave heating. Indeed, this assumption was proved correct by the chemical analyses of the regenerated samples and also when the AC was used again to adsorb phenol, a point which will be discussed later.

Sample CV presented high oxygen (9.7\%) and volatile matter (22\%) contents, compared to the Q sample (1.9 and $2.5 \%$, respectively). Volatiles and oxygen-containing functional groups were evolved during heating, the amount of gases released being much larger for sample CV than for sample Q, as might be expected. The gases evolved from the sample appeared to hinder microwave absorption, thereby impeding the heating of the AC. This fact was corroborated by: i) the large amount of transmitted power detected at the end of the microwave cavity, and ii) the maintenance of a constant temperature in the carbon bed during the process, even when the input power applied was increased. Once the volume of gases evolved had diminished (all the volatiles having already evolved) the sample was heated rapidly, while the amount of transmitted power decreased abruptly. During subsequent regeneration cycles, the CV sample did not show the same behaviour, as the volatiles and most of the oxygen content had already been desorbed in the first step (cf. Table 1). Consequently, the performance observed after the first few cycles was more similar to that of the Q sample.

These results were corroborated by the thermal analysis of the as-received activated carbons (Figure 2). The mass loss profile of the CV sample at low temperature was much more noticeable than that of the Q sample, indicating that the volatile matter of the CV sample had evolved from low temperatures. Furthermore, the proximate analysis of the CV sample showed a large volatile matter content.

\section{b) Characterization of the regenerated activated carbons}

The characteristics of the pore structure of the parent and regenerated activated carbons are presented in Table 1. The $\mathrm{N}_{2}$ adsorption isotherms at $-196{ }^{\circ} \mathrm{C}$ are shown in Figures 3 and 4 . All the adsorption isotherms are type I on the BDDT classification [26], indicating that they are mainly microporous materials. However, the desorption branch of sample CV presents a hysteresis loop at high relative pressures, pointing to a considerable development of mesoporosity. 
After the first cycle, the shape of the nitrogen isotherms moved steadily downwards, this effect being more evident for the samples regenerated in the conventional furnace. Successive cycles caused a sharp decrease in the amount of adsorbed nitrogen. After 3 and 6 regeneration cycles the porous network of the adsorbent was found to be extremely damaged. Nevertheless, dielectric heating preserved the texture to a greater extent than the conventional method.

The abrupt knee in the nitrogen adsorption isotherms of the regenerated samples indicates that the microporosity of these samples is mainly composed of pores of a small diameter. As regards mesoporosity, the hysteresis loop of the CV sample at high relative pressures appeared in all its counterparts. After six regeneration cycles, despite the significant fall in the uptake of nitrogen, mesoporosity seemed to be noticeably well preserved.

As can be seen in Table 1, there is an abrupt decrease in the surface areas of the regenerated samples. BET values (cf. Table 1) decreased considerably over the successive regeneration cycles, values being $50 \%$ lower than those of the parent samples after 3 cycles. This effect was more evident when thermal treatment was conducted in the electric furnace. For instance, the apparent BET surface area of sample Q fell by $86 \%$ after 6 cycles, compared to $76 \%$ in the microwave. These changes were even more remarkable for the Q sample than for the CV sample (except in the first cycle). The greater decrease in the BET area observed in the CV sample after the first few cycles might be associated with internal reorganizations in this activated carbon as a consequence of the large amount of gases evolved during pyrolysis. The $\mathrm{C}_{\mathrm{BET}}$ values of the regenerated samples confirmed that microporosity was constricted due to the thermal treatment of the exhausted samples, as suggested by the abrupt knee in the nitrogen adsorption isotherms. These results also prove that the adsorbate was only partially desorbed during pyrolysis. As a consequence of this partial compound removal, the porous structure of the sample remained blocked and hence the apparent BET surface area was reduced.

Thermal heating at high temperature of the non-saturated materials induced a partial collapse of the porous structure (structural annealing). This effect, which has been reported in the literature by numerous authors [27], was more significant for the CV samples.

The textural properties of the samples heated up in the absence of the adsorbate were better than those observed after the first regeneration, underlining the important role of the phenol molecules and their desorption process in the changes produced in the porous structure of the AC.

The evolution of medium-micro and mesopores was evaluated by the DFT model applied to the nitrogen adsorption isotherms (cf. Table 1). The mesopore volumes decreased substantially after the first cycle, this decrease being somewhat smaller for the following cycles. For instance, the volume of mesopores in the Q series after 6 regeneration cycles fell by $72 \%$ when the regeneration was conducted in the EF, against $62 \%$ in the microwave device. In contrast, the decrease amounted to 51 and $53 \%$ for regeneration in the EF and the microwave device, respectively, for the CV series. The medium-sized micropore volume decreased sharply as regeneration proceeded. For 
instance, regeneration of $\mathrm{Q}$ in the electric furnace gave rise to a decrease of $90 \%$ in the micropore volume compared to $82 \%$ when regeneration was conducted using microwave energy.

This suggests that the porosity of the samples was constricted as a consequence of the regeneration process. Gradual blocking of the porous structure seemed to occur because a fraction of the adsorbed molecules did not evolve from the carbon surface during regeneration. These molecules may have remained inside the pore network of the AC, and decomposed due to the high temperature of the process, giving rise to a carbonaceous residue [28-30]. The deposited carbon may have appeared as coke deposits at the entrance of the pores. The process is very similar to chemical vapour deposition, and has been widely reported in the literature [30].

The blockage, however, was less evident for the CV series, probably due to the highly developed mesoporosity of the parent sample ( 0.134 vs $0.530 \mathrm{~cm}^{3} \mathrm{~g}^{-1}$ for $\mathrm{Q}$ and $\mathrm{CV}$, respectively). Mesoporosity may permit a better connection between the microporous pattern of the activated sample (where adsorption mainly takes place), and the exterior of the carbon, permitting the molecules retained to evolve more rapidly from the carbon. In the case of the Q series, the lack of a well developed mesoporosity delayed the migration of the desorbed molecules. Consequently, the formation of coke deposits and damage to porosity was enhanced.

The micropore size distribution calculated by applying the DRS equation to the $\mathrm{CO}_{2}$ adsorption isotherms at $0{ }^{\circ} \mathrm{C}$ is shown in Figure 5. The results show a gradual decrease in the height of the maximum pore volume with the number of regeneration cycles. When regeneration was performed by conventional techniques, the decrease in micropore volume was accompanied by a downward shift to pores of narrower sizes. This suggests that regeneration treatment introduced some molecular sieve effects into the porous structure of the regenerated activated carbons, most likely due to blocking effects at the entrance of the pores of a larger size. In contrast, in the case of regeneration in the MW, a slow and gradual fall was observed and the pore width remained almost unaltered, indicating that obstruction of the pores of a larger size occurred to a lesser extent In the case of the CV series, a shift to pores of a narrow size after the first cycle and in the absence of an adsorbate was also observed, regardless of the heating mechanism employed. This can be attributed to the damage to the porous structure of the sample as a consequence of the temperature of regeneration (volatiles evolved during pyrolysis).

These results are in good agreement with those inferred from nitrogen adsorption data. If phenol molecules are not completely removed from the inner pores of the activated carbon, they decompose inside the porous structure, creating coke deposits. Thus, the microporous network of the AC is blocked and the volume of medium-sized micropores and mesopores accessible to the nitrogen probes decreases. At the same time, carbon deposition from phenol decomposition may occur at the entrance to the pores, giving rise to narrower pore size distributions, as evidenced by applying the DRS method to the $\mathrm{CO}_{2}$ adsorption data. 
The changes induced in the porous structure of the regenerated samples are due to a twofold effect; the annealing effect in the carbonaceous skeleton when the samples are exposed to high temperatures, and the creation of coke deposits within the pore structure [28-30], as a consequence of which the surface area decreases and the micropore distribution shifts towards narrower pores, regardless of the experimental device employed.

Textural properties are preserved to a greater extent in the case of microwave energy. This might be due to the different heating mechanisms in both devices, along with the shorter exposure time at the temperature of regeneration in the microwave system. In the microwave, due to the shorter exposure time, the AC were less prone to suffer structural porous blockage.

Whereas heating by conventional means occurs by convection, microwaves heat the samples from the inside, creating a thermal temperature gradient that decreases towards the surface of the material [31]. Due to this temperature gradient, there is a heat flow from the inside of the particle (hot area) to the outside (cool area), unlike the case of conventional heating. Consequently, the desorbed molecules produced in the core of the carbon bed diffuse towards the lower temperature region more quickly.

Since desorption occurs at the surface of the carbon bed, the phenol molecules inside the AC have to migrate to the surface. As the diffusion towards the surface is the rate-determining step in the process, the desorption process is favoured during microwave irradiation.

While the desorbed molecules are being transferred through the carbon bed towards the surface, unwanted parallel reactions such as the decomposition of the evolved molecules may occur. These reactions were unfavored in the microwave device, due to the short exposure time and the temperature gradient which enhanced the desorption process. Therefore, the inner porous structure of the samples, which would otherwise have been destroyed, was better preserved.

In the conventional method, however, the AC is heated by convective heat transfer from a high-temperature gas and by conduction from the surface to the centre, with a temperature gradient that is in the inverse direction to that of microwave heating; i.e., a lower temperature in the centre and a higher one in the outer region.

There is a point in conventional heating where the molecules have substantially evolved, but there remains an exhausted core. Transmission of heat to this inner core is slower by conventional techniques, so that desorption from this central core is more difficult. As regeneration develops from the surface to the centre, the desorbed molecules produced near the centre have to traverse a high temperature surface region. For this reason, it is difficult to prevent the decomposition of the desorbed molecules inside the porous network of the activated carbon. This causes the physical rupture of the pore walls and a greater obstruction of the pores, compared to the samples regenerated with microwave energy (cf. Table 1). 
It is well-known that thermal treatment of activated carbons decreases their oxygen content. Most of the oxygen is originally present in the form of functional groups located around the edges of the carbon layer planes. As a consequence of the regeneration temperature, the oxygenated functional groups of the $\mathrm{CV}$ sample are evolved as $\mathrm{CO}$ and $\mathrm{CO}_{2}$ during the process. The large amount of gases evolved from the CV sample, compared to the Q sample, is due to its high oxygen and volatile contents. Furthermore, the PZC values (Table 1) showed that the regeneration treatment gave rise to highly basic materials, regardless of the heating mechanism. This effect is much more noticeable for the CV sample, which initially showed an acidic nature. Most of the functional groups of the CV sample were eliminated during the first and second regeneration cycles, as the PZC values after the third regeneration cycle were of a basic nature.

The results of the loading capacity of phenol on the parent and regenerated activated carbons are presented in Table 2. The adsorptive capacities of the regenerated samples were greater when dielectric heating was employed in the regeneration process. There are two reasons for this behaviour: firstly, the more developed textural properties of the samples regenerated with microwave energy, and secondly, the changes induced in the surface chemistry after regeneration.

Using conventional heating (EF) the adsorption capacity of the AC was observed to decrease gradually with subsequent regeneration cycles, this effect being more evident for the CV series. This decrease is in agreement with the partial desorption of phenol molecules and the blockage of the porous structure. As the porosity diminished, the adsorption capacity also decreased, despite the increasing basicity of the samples (cf. Table 1).

In contrast, it is worth noting that after one regeneration cycle, despite the slight deterioration of the porous structure of the parent activated carbons -compared to the EF furnace-, adsorptive capacities after MW regeneration were unexpectedly high. The loading capacity of phenol exceeded its initial value, particularly for the CV sample.

Adsorptive capacities after the second and even the third regeneration cycles in the MW device were also significantly high. Afterwards, the adsorptive capacities slowly decreased. For instance, after the first cycle the phenol adsorption capacity in the Q sample was $425 \mathrm{mg} \mathrm{g}^{-1}$ in the MW, compared to $161 \mathrm{mg} \mathrm{g}^{-1}$ in the electric furnace. After 6 cycles, the decrease in adsorption capacity compared to the initial sample was $70 \%$ in the MW versus $82 \%$ in the EF. Thus it can be inferred that microwave energy contributes to an increase in the efficiency of the regeneration of saturated $\mathrm{AC}$, and in the life span of the activated carbon.

This behaviour can be explained in terms of textural changes and surface chemistry. Since thermal treatment of the activated carbons eliminated the oxygenated surface functional groups, the $\pi$-electron density of the graphene layers increased, as did the dispersive interactions between the phenol and the $\pi$-electron density of the carbon [32-34]. Accordingly, there was an increase in the 
chemisorption contribution to the overall loading capacity of phenol, this capacity increasing extensively after the first regeneration cycle. In the case of the CV series, the increase in the contribution of chemisorption might be expected to be more pronounced, due to the primary acidic nature of this AC. In fact, deterioration of the textural properties due to the high amount of gases evolved during heating at high temperature restricted the increase in the overall uptake of phenol.

\section{CONCLUSION}

Dielectric heating of activated carbons was found to be more rapid and efficient than convection methods, although the input power of the microwaves needed to be increased when the samples were saturated. After the first regeneration cycle, any further increase in the regeneration/adsorption step meant an increase of double the amount of power applied. Nevertheless, the main advantage of using microwave energy is that the treatment can be performed in a relatively short period of time, which implies a lower consumption of gas and energy and a process that is still economically attractive and feasible.

The changes induced in the porous structure of the regenerated samples are due to a twofold effect; on the one hand the partial collapse of porosity due to the thermal heating itself, and secondly the formation of coke deposits within the pore network as a consequence of the decomposition of phenol molecules that did not evolve.

When microwave heating was applied a temperature gradient was created in the samples, which decreased from the core towards the surface of the material. Thus, any desorbed molecules migrated rapidly to the surface, the latter having a lower temperature than the core of the sample. This reduced the formation of coke deposits inside the porous structure of the AC, resulting in a better preservation of the textural characteristics of the activated carbon.

In the conventional heating, the desorbed molecules produced near the centre decomposed inside the porous network of the activated carbon, before they could be completely evolved from the carbon surface. Thus, coke residues blocked the pore structure of the adsorbent, leading to a greater decrease in the apparent BET surface areas and micropore volumes.

Adsorptive capacities were found to be much higher when regeneration was carried out in the microwave device, even after various cycles of regeneration, compared to those of the conventional furnace. This is attributed mainly to the changes induced in the surface chemistry and textural properties of the regenerated samples.

\section{ACKNOWLEDGEMENT}


Work carried out with a financial grant from the European Coal and Steel Community (Project 7220-PR-139). The authors wish to thank Dr. Martín-Gullón for his considerate and helpful assistance with the software tool used for the DRS calculation.

\section{REFERENCES}

[1] K.D. Henning, S. Schäfer, Gas Sep. Pur. 74 (1993) 235.

[2] D.W. Mazyck, F.S. Cannon, Carbon 38 (2000) 1785.

[3] G.M. Walker, L.R. Weatherley, Sep. Sci. Technol. 35 (2000) 1329.

[4] M. Gurrath, T. Kuretzky, H.P. Boehm, L.B. Okhlopkova, A.S. Lisitsyn, V.A. Likholobov, Carbon 38 (2000) 1241.

[5] Roskill Information Services Ltd. The economics of activated carbon. Clapham Road. SW9 OJA, London, 1998, p. 17.

[6] I. Dranca, Y. Lupascu, K. Vogelsang, L. Monahova, J. Therm. Anal. 34 (2001) 945.

[7] M. Sheintuch, Y.I. Matatov-Meytal, Catal. Today 53 (1999) 73.

[8] D.W. Price, P.S. Schmidt, J. Microw. Power Electromagnetic Energy 32 (1997) 145.

[9] Y. Kong, C.Y. Cha, Energy and Fuels 10 (1996) 1245.

[10] S.M. Bradshaw, E.J. van Wyk, J.B. De Swardt, J. Microwave Power Electromagnetic Energy 32 (1997) 131.

[11] S.M. Bradshaw, E.J. van Wyk, J.B. Swardt, J. South African Inst. Minning Metall. 4 (1998) 201.

[12] P.M. Coss, C.Y. Cha, J. Air Waste Management Assoc. 50 (2000) 529.

[13] I.S. Balba, S.J. Oda, K.E. Haque, P.D. Kondos, R.J.C. MacDonald, Ceramic Transactions. The American Ceramic Society 21 (1991) 475.

[14] C.Y. Cha, M.W. Carlisle, J. Air Waste Management Assoc. 51 (2001) 1628.

[15] D.A. Jones, T.P. Lelyveld, S.D. Mavrofidis, S.W. Kingman, N.J. Miles, Resources, Conservation and Recycling 34 (2002) 75.

[16] H.S. Ku, E. Siores, A. Taube, J.A.R. Ball, Computers and Industrial Engineering 42 (2002) 281.

[17] C.O. Ania, J.A. Menéndez, J.B. Parra, J.J. Pis, Carbon 42 (2004) 1377.

[18] Olivier, J., Carbon 36 (1998) 1469. 
[19] T.J. Bandosz, M.J. Biggs, K.E. Gubbins, Y. Hattori, Y. Liyama, K. Kaneko, J. Pikunic, K.T. Thomson, in Chemistry and Physics of Carbons, vol 28, Marcel \& Dekker, New York, 2003, p 41.

[20] J.F. Byrne, H. Marsh, in Porosity in carbons; characterization and applications, Ed. Patrick JW, Edward Arnold, Great Britain, 1995, p. 1.

[21] A. Guillot, F. Stoeckli, Carbon, 39 (2001) 2059.

[22] J. Garrido, A. Linares-Solano, J.M. Martín-Martínez, M. Molina-Sabio, F. Rodríguez-Reinosos, R. Torregrosa, Langmuir, 3 (1987) 76.

[23] J.S. Noh, A. Shwarz, J. Colloid Interf. Sci. 142 (1992) 233.

[24] K.E. Haque, Int. J. Miner Process 57 (1999) 1.

[25] A. Zlotorzynski, Critic Rev. Anal. Chem. 25 (1995) 43.

[26] S. Brunauer, L.S. Deming, W.E. Deming, E. Teller, J. Am. Chem. Soc., 62 (1940) 1723.

[27] A.M. Rao, A.W.P. Fung, M.S. Dresselhaus, Endo M, J. Mater. Res. 7 (1996) 1788.

[28] S. Cooke, M.M. Labes, Carbon 32 (1994) 1055.

[29] C. Moreno-Castilla, J. Rivera-Utrilla, J.P. Joly, M.V. López-Ramón, M.A. Ferro-Garcia, F. Carrasco-Marin, Carbon, 33 (1995) 1417.

[30] S. I. Ayuthaya, N. Mongkolsiri, P. Praserthdam, P.L. Silveston, Applied Catalysis B: Environmental 43 (2003) 1-12

[31] M. Miura, H. Kaga, A.Sakurai, T. Kakuchi, K. Takahashi, J. Anal. Appl. Pyrolysis 71 (2004) 187.

[32] D.M. Nevskaia, A. Santianes, V. Muñoz, A. Guerrero Ruiz, Carbon 37 (1999) 1065.

[33] C. Moreno-Castilla, J. Rivera-Utrilla, M.V. López-Ramón, F. Carrasco-Marín, Carbon 33 (1995) 845.

[34] L.R. Radovic, I.F. Silva, J.L. Ume, J.A. Menéndez, C.A. Leon, A.W. Scaroni, Carbon 9 (1997) 1339. 


\section{List of Tables}

Table 1. Chemical and textural properties of the parent and regenerated samples, from $\mathrm{N}_{2}$ adsorption isotherms at $-196^{\circ} \mathrm{C}$, using the BET and DFT methods.

Table 2. Adsorption capacities of the as-received and the thermally regenerated samples

\section{Figure captions}

Figure 1. Variation in the input power applied during the regeneration assays in the microwave device.

Figure 2. TG curves of the as-received activated carbons.

Figure 3. $\mathrm{N}_{2}$ adsorption isotherms at $-196^{\circ}$ corresponding to the as-received and the regenerated samples after 1,3 and 6 regeneration cycles.

Figure $4 . \mathrm{N}_{2}$ adsorption isotherms at $-196{ }^{\circ} \mathrm{C}$ corresponding to the samples treated in the absence of adsorbate.

Figure 5. Micropore size distribution obtained from the DRS method applied to the $\mathrm{CO}_{2}$ adsorption isotherms at $0{ }^{\circ} \mathrm{C}$ 
Table 1. Chemical and textural properties of the parent and regenerated samples, from $\mathrm{N}_{2}$ adsorption isotherms at $-196^{\circ} \mathrm{C}$, using the BET and DFT methods.

\begin{tabular}{|c|c|c|c|c|c|c|c|}
\hline & \multicolumn{2}{|c|}{ BET method } & \multicolumn{3}{|c|}{ DFT method } & \multirow[b]{2}{*}{$\begin{array}{c}\text { Oxygen } \\
(\%)\end{array}$} & \multirow[b]{2}{*}{$\begin{array}{l}\mathbf{p H} \\
\mathbf{P Z C}\end{array}$} \\
\hline & $\begin{array}{c}S_{\text {BET }} \\
\left(\mathrm{m}^{2} \mathrm{~g}^{-1}\right)\end{array}$ & $\mathrm{C}_{\text {BET }}$ & $\begin{array}{c}V_{\text {narrow }} \\
\text { micropores } \\
\left(\mathrm{cm}^{3} \mathbf{g}^{-1}\right)\end{array}$ & $\begin{array}{l}V_{\text {medium- }} \\
\text { micropores } \\
\left(\mathrm{cm}^{3} \mathbf{g}^{-1}\right)\end{array}$ & $\begin{array}{c}V_{\text {mesopores }} \\
\left(\mathrm{cm}^{3} \mathrm{~g}^{-1}\right)\end{array}$ & & \\
\hline $\mathbf{Q}$ & 1149 & 262 & 0.126 & 0.211 & 0.134 & 1.9 & 9.2 \\
\hline Q EF & 1022 & 353 & 0.136 & 0.179 & 0.065 & 1.1 & 9.8 \\
\hline QC1R1 EF & 822 & 302 & 0.119 & 0.129 & 0.071 & 1.2 & 10.3 \\
\hline QC3R3 EF & 340 & 130 & 0.024 & 0.063 & 0.051 & 1.0 & 9.5 \\
\hline QC6R6 EF & 162 & 88 & 0.002 & 0.024 & 0.037 & 1.0 & 9.3 \\
\hline Q MW & 1083 & 309 & 0.131 & 0.192 & 0.101 & 1.4 & 10.8 \\
\hline QC1R1 MW & 932 & 289 & 0.111 & 0.166 & 0.087 & 0.9 & 10.2 \\
\hline QC3R3 MW & 595 & 145 & 0.052 & 0.085 & 0.068 & 0.9 & 9.5 \\
\hline QC6R6 MW & 257 & 105 & 0.021 & 0.037 & 0.051 & 0.9 & 9.3 \\
\hline $\mathrm{CV}$ & 1414 & 157 & 0.112 & 0.243 & 0.530 & 9.7 & 2.2 \\
\hline CV EF & 961 & 287 & 0.142 & 0.105 & 0.347 & 6.8 & 3.5 \\
\hline CVC1R1 EF & 908 & 273 & 0.137 & 0.099 & 0.330 & 6.8 & 3.6 \\
\hline CVC3R3 EF & 525 & 248 & 0.066 & 0.052 & 0.275 & 4.2 & 7.3 \\
\hline CVC6R6 EF & 374 & 159 & 0.038 & 0.035 & 0.249 & 2.4 & 8.7 \\
\hline CV MW & 1062 & 297 & 0.142 & 0.163 & 0.462 & 6.1 & 4.2 \\
\hline CVC1R1 MW & 961 & 268 & 0.132 & 0.116 & 0.352 & 5.7 & 4.5 \\
\hline CVC3R3 MW & 547 & 265 & 0.023 & 0.056 & 0.286 & 4.0 & 7.1 \\
\hline CVC6R6 MW & 386 & 175 & 0.018 & 0.043 & 0.262 & 1.9 & 9.2 \\
\hline
\end{tabular}


Table 2. Adsorption capacities of the as-received and the thermally regenerated samples

\begin{tabular}{|lcc|lcc|}
\hline \multicolumn{7}{|c|}{ Adsorptive capacities $\left(\mathbf{m g ~ g}^{-\mathbf{1}}\right)$} \\
\hline Q & 226 & MW & EF & MW & EF \\
\hline & EF & 425 & CVC1R1 & 210 & 238 \\
\hline QC1R1 & 161 & 307 & CVC2R2 & 167 & 265 \\
QC2R2 & 113 & 115 & CVC3R3 & 147 & 157 \\
QC3R3 & 71 & 82 & CVC4R4 & 91 & 104 \\
QC4R4 & 55 & 70 & CVC5R5 & 88 & 96 \\
QC5R5 & 40 & 59 & CVC6R6 & 65 & 83 \\
QC6R6 & 32 & & & & \\
\hline
\end{tabular}


Figure 1. Variation in the input power applied during the regeneration assays in the microwave device.

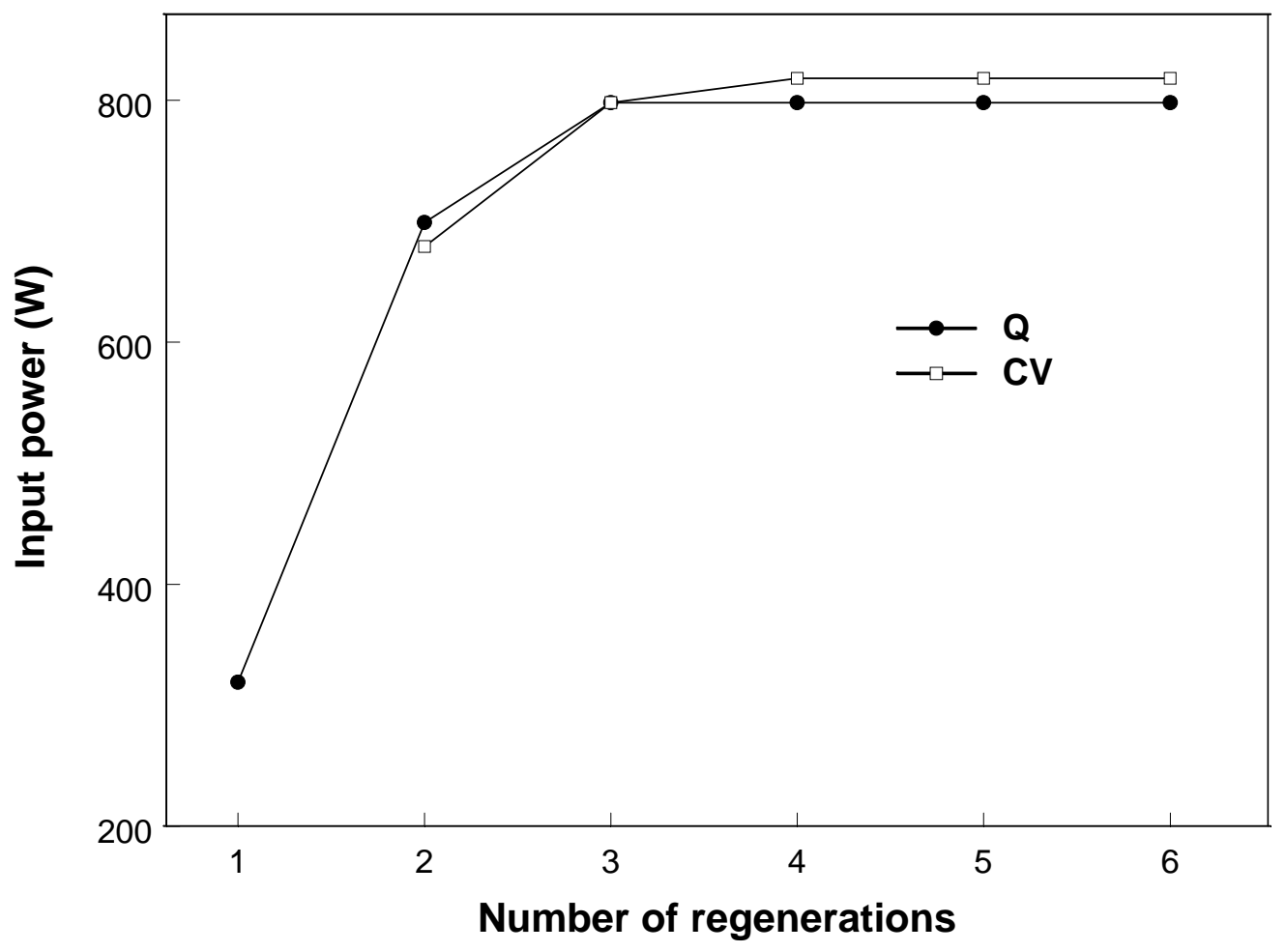


Figure 2. TG curves of the as-received activated carbons.

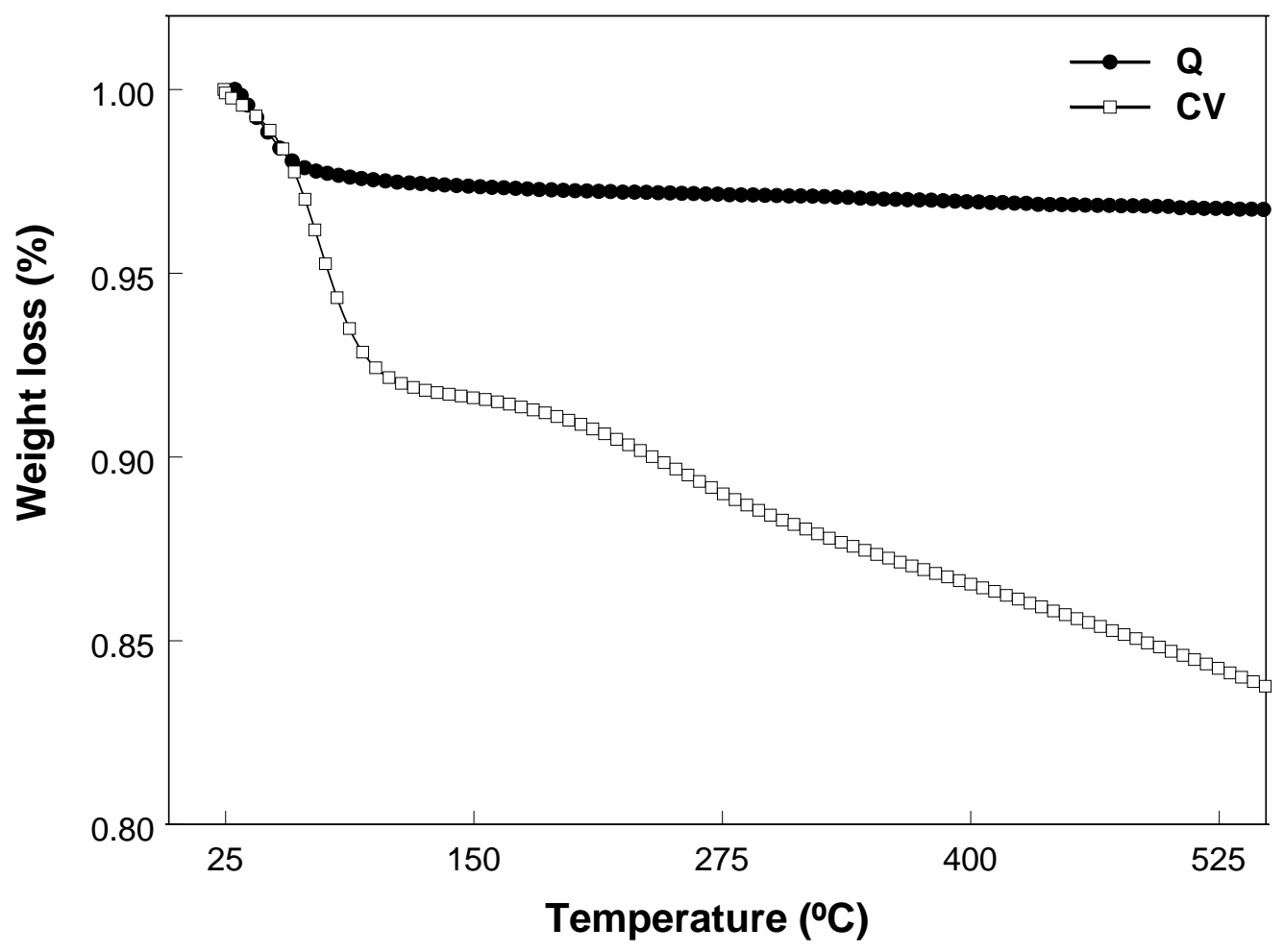


Figure 3. $N_{2}$ adsorption isotherms at $-196^{\circ}$ corresponding to the as-received and the regenerated samples after 1, 3 and 6 regeneration cycles.
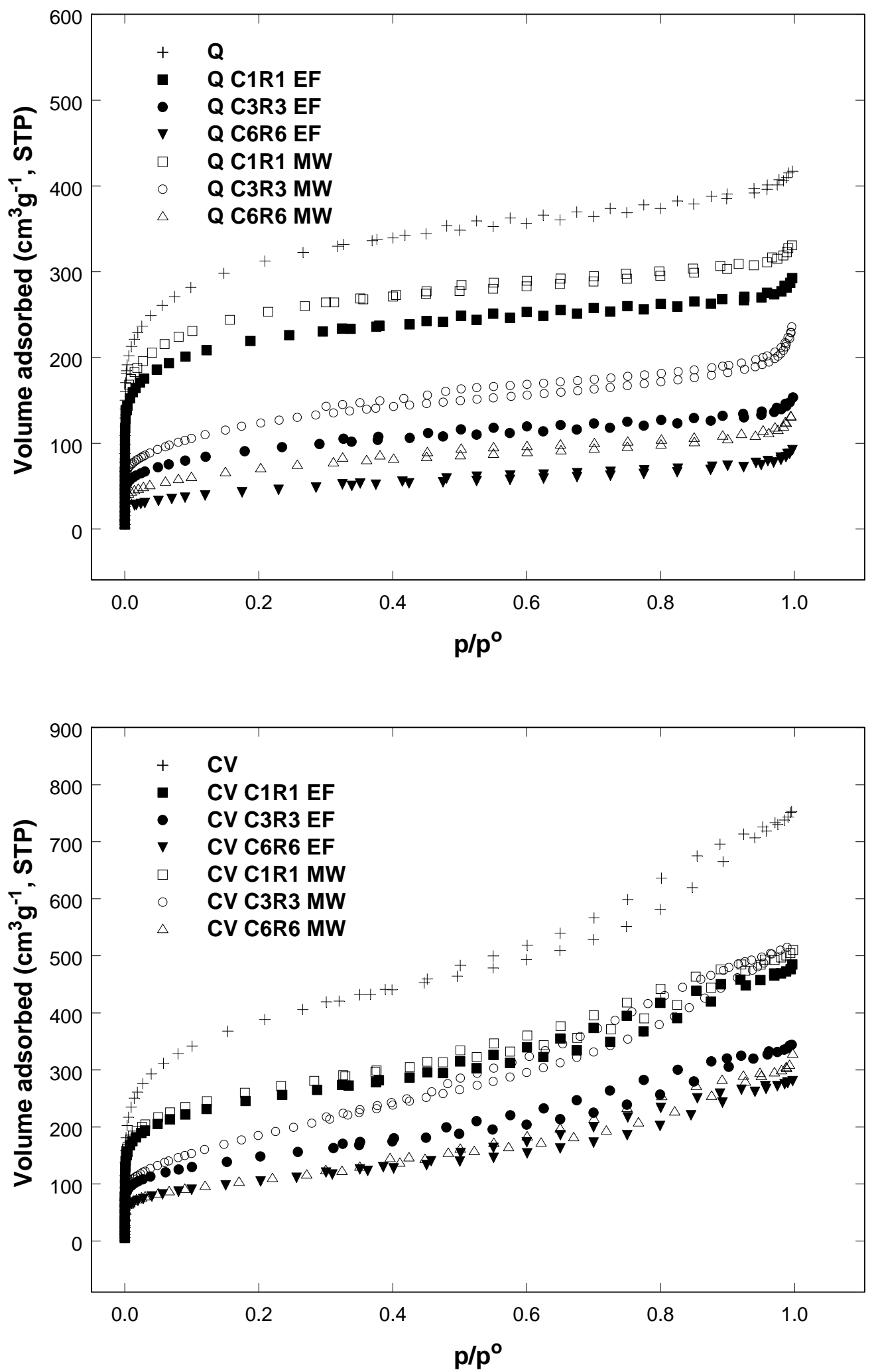
Figure 4. $N_{2}$ adsorption isotherms at $-196{ }^{\circ} \mathrm{C}$ corresponding to the samples treated in the absence of adsorbate.
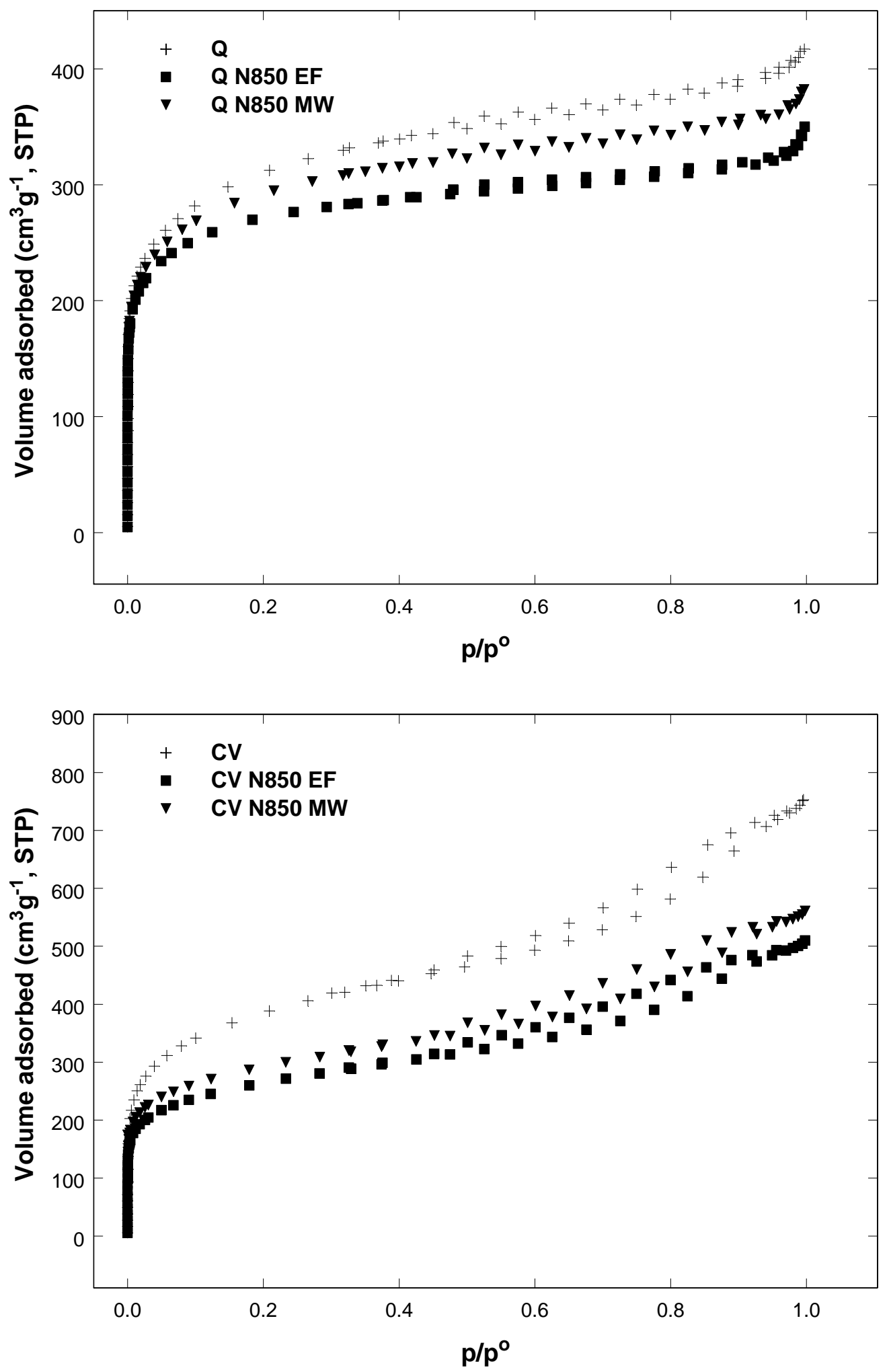
Figure 5. Micropore size distribution obtained from the DRS method applied to the $\mathrm{CO}_{2}$ adsorption isotherms at $0^{\circ} \mathrm{C}$.
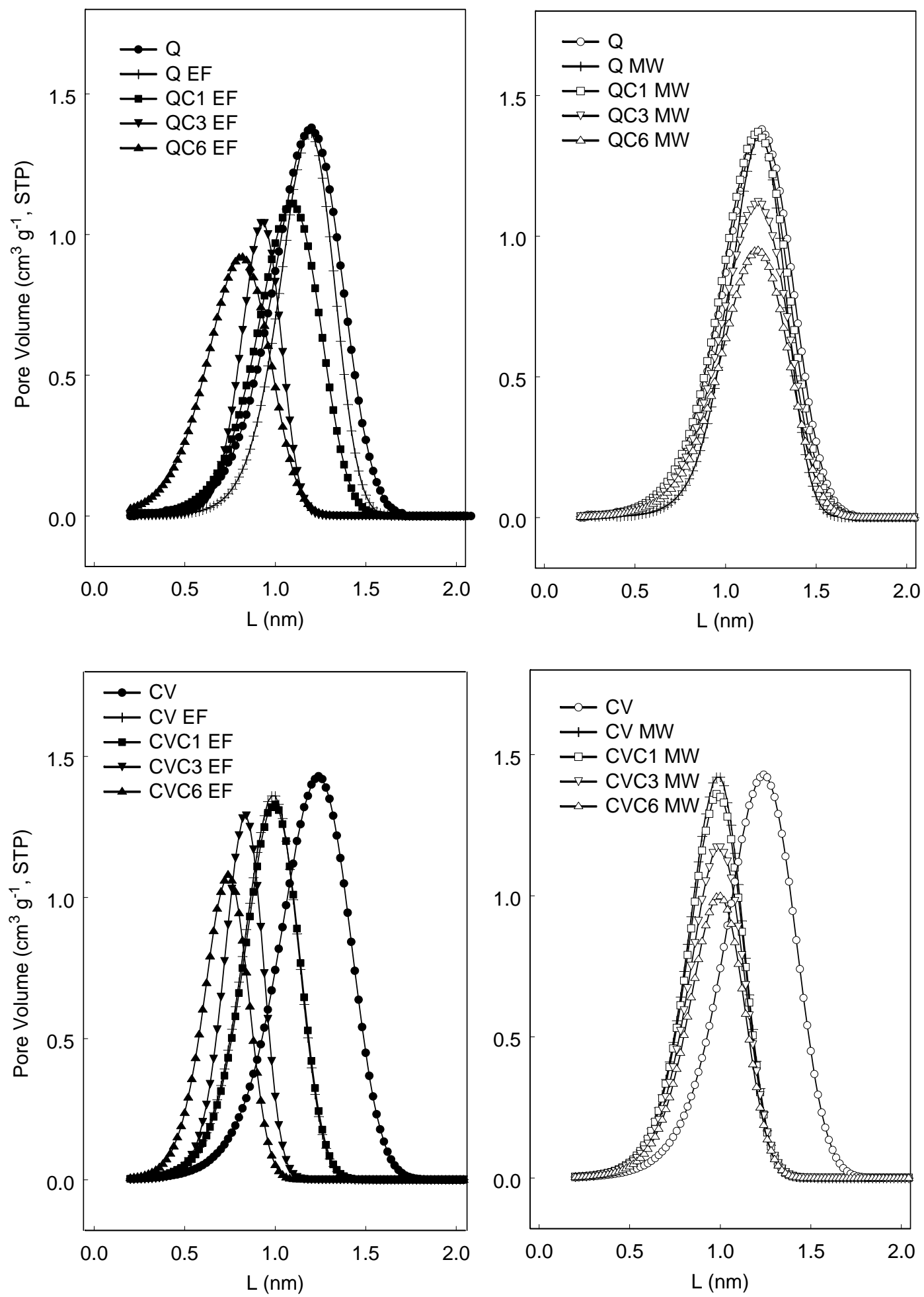